\title{
REGULAR SETS OF SAMPLING AND INTERPOLATION FOR WEIGHTED BERGMAN SPACES
}

\author{
KRISTIAN SEIP
}

(Communicated by Theodore W. Gamelin)

\begin{abstract}
Let $z_{m n}=a^{m}(b n+i), a>1, b>0, m, n$ integers. For each weighted Bergman space on the upper half-plane there exists a constant $c>0$ such that $\left\{z_{m n}\right\}$ is a set of sampling if and only if $b \ln a<c$ and a set of interpolation if and only if $b \ln a>c$. When $b \ln a=c,\left\{z_{m n}\right\}$ is a set of uniqueness.
\end{abstract}

\section{INTRODUCTION}

Inspired by the work of Beurling [1, pp. 341-365], Landau investigated in [7] sets of sampling and interpolation for a class of entire functions containing as a subclass the Paley-Wiener space of bandlimited functions. This study revealed in a precise way the role of the Nyquist density in information theory. In a recent paper [11] $R$. Wallstén and the author showed that sets of sampling and interpolation for the Bargmann-Fock space can (as for the Paley-Wiener space) be described in terms of a certain critical density, which again can be interpreted as the Nyquist density. [11] confirmed in particular a conjecture made by Daubechies and Grossmann [4] about the special role of the von Neumann lattice.

The results of this paper yield the first evidence that a critical quantity similar to the Nyquist density exists for Bergman spaces. More specifically, we will detect a discrete set that relates to the weighted Bergman space exactly as the von Neumann lattice does to the Bargmann-Fock space.

In order to state our results in a precise manner, let $U=\{z=x+i y: y>0\}$ denote the upper half-plane, and define for each $\alpha>1$ the weighted Bergman space,

$$
A_{\alpha}(U)=\left\{f \text { analytic in } U: \iint_{U}|f(z)|^{2} y^{\alpha-2} d x d y<\infty\right\} .
$$

We say that a discrete subset $\left\{z_{j}\right\}$ of $U$ is a set of sampling for $A_{\alpha}(U)$ if there exist positive constants $A$ and $B$ such that

$$
A \iint_{U}|f(z)|^{2} y^{\alpha-2} d x d y \leq \sum_{j}\left|f\left(z_{j}\right)\right|^{2} y_{j}^{\alpha} \leq B \iint_{U}|f(z)|^{2} y^{\alpha-2} d x d y
$$

Received by the editors April 4, 1991 and, in revised form, May 30, 1991.

1991 Mathematics Subject Classification. Primary 30D10, 30E05, 46E20; Secondary 81R30. 
for every $f \in A_{\alpha}(U) .\left\{z_{j}\right\}$ is a set of interpolation for $A_{\alpha}(U)$ if for every sequence $\left\{a_{j}\right\}$ for which $\left\{y_{j}^{\alpha / 2} a_{j}\right\} \in l^{2}$, there exists a function $f \in A_{\alpha}(U)$ such that $f\left(z_{j}\right)=a_{j}$ for all $j$. For given parameters $a>1, b>0$ we define the (regular) discrete set $\Gamma(a, b)=\left\{z_{m n}\right\}_{m, n \in Z}$, where $z_{m n}=a^{m}(b n+i)$. Our main results can then be stated in terms of the following two theorems.

Theorem 1.1. $\Gamma(a, b)$ is a set of sampling for $A_{\alpha}(U)$ if and only if $b \ln a<$ $4 \pi /(\alpha-1)$.

Theorem 1.2. $\Gamma(a, b)$ is a set of interpolation for $A_{\alpha}(U)$ if and only if $b \ln a>$ $4 \pi /(\alpha-1)$.

It will follow easily from our method of proof that for the critical value $b \ln a=4 \pi /(\alpha-1), \Gamma(a, b)$ is a set of uniqueness for $A_{\alpha}(U)$; this means that if $f\left(z_{m n}\right)=0$ for all $z_{m n}$, then $f \equiv 0$.

In the last section we have included a brief remark on the physical interpretation of weighted Bergman spaces, because this aspect has been an important motivation for our work. For general background on Bergman spaces one may consult [12].

\section{A SPECIAL FUNCTION}

In this section we construct a function that will play the role of the sine in the Paley-Wiener space and that of the Weierstrass $\sigma$-function in the BargmannFock space. We obtain precise bounds on its growth and, equipped with these estimates, we will succeed in employing the ideas of [11] in the next section.

To $\Gamma(a, b)$ we associate the expression

$$
h(z)=\left(\prod_{k=0}^{\infty} \frac{\sin \pi b^{-1} a^{-k}\left(i a^{k}-z\right)}{\sin \pi b^{-1} a^{-k}\left(i a^{k}+z\right)}\right)\left(\prod_{m=1}^{\infty} e^{2 \pi b^{-1}} \frac{\sin \pi b^{-1} a^{m}\left(z-i a^{-m}\right)}{\sin \pi b^{-1} a^{m}\left(z+i a^{-m}\right)}\right) .
$$

By applying the usual convergence test for infinite products, we find that the two products converge absolutely for every $z=x+i y, y>0$, and $h(z)$ is thus a function analytic in the upper half-plane with $\Gamma(a, b)$ as its zero-set.

We check directly that $h(z)$ has the basic property

$$
h(a z)=-e^{-2 \pi b^{-1}} h(z) \text {. }
$$

This relation can be used to estimate the growth of $h$ in the following way. First a straightforward computation shows that there exist positive constants $C_{1}$ and $C_{2}$ so that

$$
C_{1} \rho_{U}(z, \Gamma(a, b)) \leq|h(z)| \leq C_{2}
$$

in the strip $a^{-1 / 2} \leq y \leq a^{1 / 2}$, where $\rho_{U}(\cdot, \cdot)$ denotes the pseudohyperbolic distance on $U$,

$$
\rho_{U}(z, \zeta)=\left|\frac{z-\zeta}{z-\bar{\zeta}}\right|
$$

Combining these inequalities with (1) and the fact that $\rho_{U}(a z, \Gamma(a, b))=$ $\rho_{U}(z, \Gamma(a, b))$, we obtain the global estimates

$$
|h(z)| \leq C y^{-2 \pi(b \ln a)^{-1}}
$$

and

$$
|h(z)| \geq C \rho_{U}(z, \Gamma(a, b)) y^{-2 \pi(b \ln a)^{-1}} .
$$


For simplicity, we put $\beta=2 \pi(b \ln a)^{-1}$ in the sequel.

We note in passing that (1) shows that $h(z)$ is an automorphic form with respect to the Fuchsian group generated by the transformation $z \mapsto a z$.

Next we construct a related function in the unit disk $D=\{z:|z|<1\}$,

$$
g(z)=(1-z)^{-2 \beta} h\left(i \frac{1+z}{1-z}\right) .
$$

From (2) and (3) we obtain immediately the estimates

$$
|g(z)| \leq C\left(1-|z|^{2}\right)^{-\beta}
$$

and

$$
|g(z)| \geq C \rho_{D}(z, \Lambda)\left(1-|z|^{2}\right)^{-\beta},
$$

where now $\rho_{D}(\cdot, \cdot)$ denotes the pseudohyperbolic distance on $D$,

$$
\rho_{D}(z, \zeta)=\left|\frac{z-\zeta}{1-\bar{\zeta} z}\right|
$$

and $\Lambda=\left\{z_{j}\right\}$ is the image of $\Gamma(a, b)$ under the inverse Cayley transform. From (5) we get

$$
\left|g^{\prime}\left(z_{j}\right)\right| \geq C\left(1-\left|z_{j}\right|^{2}\right)^{-\beta-1}
$$

which also will be needed.

\section{Proofs}

It will be most convenient for us to carry out the proofs in the unit disk. We then make use of the fact that

$$
f \mapsto(z+i)^{-2 \alpha} f\left(\frac{z-i}{z+i}\right)
$$

is, up to a constant multiple, a unitary map from

$$
A_{\alpha}(D)=\left\{f \text { analytic in } D: \iint_{D}|f(z)|^{2}(1-|z|)^{\alpha-2} d x d y<\infty\right\}
$$

to $A_{\alpha}(U) .\left\{z_{j}\right\}$ is a set of sampling for $A_{\alpha}(D)$ if there exist positive constants $A$ and $B$ such that

$$
\begin{aligned}
A \iint_{D}\left(1-|z|^{2}\right)^{\alpha}|f(z)|^{2} d \mu(z) & \leq \sum_{j}\left(1-\left|z_{j}\right|^{2}\right)^{\alpha}\left|f\left(z_{j}\right)\right|^{2} \\
& \leq B \iint_{D}\left(1-|z|^{2}\right)^{\alpha}|f(z)|^{2} d \mu(z)
\end{aligned}
$$

for every $f \in A_{\alpha}(D)$; here and in the sequel $\mu$ denotes the invariant area measure of $D$ with $d \mu(z)=\left(1-|z|^{2}\right)^{-2} d x d y .\left\{z_{j}\right\}$ is a set of interpolation for $A_{\alpha}(D)$ if for every sequence $\left\{a_{j}\right\}$ for which $\left\{\left(1-\left|z_{j}\right|^{2}\right)^{\alpha / 2} a_{j}\right\} \in l^{2}$, there exists a function $f \in A_{\alpha}(D)$ such that $f\left(z_{j}\right)=a_{j}$ for all $j$. These definitions are equivalent to the ones made for $A_{\alpha}(U)$ when sets in $D$ are associated to sets in $U$ via the Cayley transform.

The following simple fact will be used repeatedly. Suppose for a moment, quite generally, that $\left\{z_{j}\right\}$ is a uniformly discrete set, i.e., we assume that

$$
\inf _{j \neq k} \rho_{D}\left(z_{j}, z_{k}\right)>0 \text {. }
$$


Then if $f$ is analytic in $D$, we have

$$
\sum_{j}\left(1-\left|z_{j}\right|^{2}\right)^{s}\left|f\left(z_{j}\right)\right|^{2} \leq C \iint_{D}\left(1-|z|^{2}\right)^{s}|f(z)|^{2} d \mu(z)
$$

whenever $s>1$. This is easy to see, e.g., as a consequence of the CauchySchwarz inequality and the reproducing formula [10]

$$
f(z)=C(\delta, s) \iint_{\rho_{D}(z, \zeta)<\delta}(1-\bar{\zeta} z)^{-s} f(\zeta)\left(1-|\zeta|^{2}\right)^{s} d \mu(\zeta) .
$$

Equation (8) applies in particular to $\Lambda$, which obviously is a uniformly discrete set.

The main tool in the proof of Theorem 1.1 will be the following interpolation formula.

Lemma 3.1. If $\beta>(\alpha-1) / 2$ and $s$ is any real number, we have for each $f \in A_{\alpha}(D)$,

$$
f(z)=\sum_{j} \frac{f\left(z_{j}\right)}{g^{\prime}\left(z_{j}\right)} \frac{g(z)}{\left(z-z_{j}\right)}\left(\frac{1-|z|^{2}}{1-\bar{z} z_{j}}\right)^{s},
$$

with uniform convergence on compact sets.

Proof. By the Cauchy-Schwarz inequality and (6) we have

$$
\begin{aligned}
& \left|\sum_{j} \frac{f\left(z_{j}\right)}{g^{\prime}\left(z_{j}\right)} \frac{g(z)}{\left(z-z_{j}\right)}\left(\frac{1-|z|^{2}}{1-\bar{z} z_{j}}\right)^{s}\right|^{2} \\
& \quad \leq C \sum_{j}\left|f\left(z_{j}\right)\right|^{2}\left(1-\left|z_{j}\right|^{2}\right)^{\alpha} \sum_{k}\left(1-\left|z_{k}\right|^{2}\right)^{2+2 \beta-\alpha}\left|\frac{g(z)}{z-z_{k}}\right|^{2}\left|\frac{1-|z|^{2}}{1-\bar{z} z_{k}}\right|^{2 s},
\end{aligned}
$$

so that by (8), the sum converges for each $f \in A_{\alpha}(D)$ since $2+2 \beta-\alpha>1$.

Let $d>0$ be a number such that the circles $\rho_{D}\left(z, z_{j}\right)=d$ are nonintersecting. We let $S(r)$ be the closed path consisting of those $z$ on the circle $|z|=r$ for which $\rho_{D}(z, \Lambda) \geq d$ and of the shorter parts of the circles $\rho_{D}\left(z, z_{j}\right)=d$ that intersect the circle $|z|=r$. It is clear that $|S(r)|$ is bounded. Let $\Omega(r)$ denote the domain bounded by $S(r)$ and let $f$ be any polynomial. For $z \in \Omega(r) \backslash \Lambda$ we have by the calculus of residues that

$\frac{1}{2 \pi i} \int_{S(r)} \frac{f(\zeta)}{(1-\bar{z} \zeta)^{s} g(\zeta)} \frac{d \zeta}{\zeta-z}=\frac{f(z)}{\left(1-|z|^{2}\right)^{s} g(z)}-\sum_{z_{j} \in \Omega(r)} \frac{f\left(z_{j}\right)}{\left(1-\bar{z} z_{j}\right)^{s} g^{\prime}\left(z_{j}\right)} \frac{1}{z-z_{j}}$.

$\rho_{D}(S(r), \Lambda) \geq d$ and (5) imply that the integral on the left-hand side tends to 0 as $r \rightarrow 1$. This proves the formula for polynomials, and since the polynomials are dense in $A_{\alpha}(D)$, it is clear by (10) and (8) that the series converges to $f(z)$ for all $f \in A_{\alpha}(D)$. 
We will also need

Lemma 3.2. For $1<s<t$, we have

$$
\iint_{D} \frac{\left(1-|z|^{2}\right)^{s}}{|1-\bar{\zeta} z|^{t}} d \mu(z) \leq C\left(1-|\zeta|^{2}\right)^{s-t}
$$

and

$$
\sum_{j} \frac{\left(1-\left|z_{j}\right|^{2}\right)^{s}}{\left|1-\bar{\zeta} z_{j}\right|^{t}} \leq C\left(1-|\zeta|^{2}\right)^{s-t}
$$

for any uniformly discrete set $\left\{z_{j}\right\}$.

Equation (11) is contained in Lemma 4.2.2 in [12, p. 53], and (12) follows from (11) by (8).

Before proving the two theorems, we indicate how to show that $\Gamma(a, b)$ is a set of uniqueness when $b \ln a=4 \pi /(\alpha-1)$. If $f\left(z_{j}\right)=0$ for all $j$, we may write $f(z)=\tilde{f}(z) g(z)$ for some function $\tilde{f}$ analytic in $D$. By (5) and an argument similar to that of the proof of Proposition 3.1 in [11], $f \in A_{\alpha}(D)$ implies

$$
\iint_{D}|\tilde{f}(z)|^{2}\left(1-|z|^{2}\right)^{-1} d x d y<\infty
$$

which is absurd unless $\tilde{f} \equiv 0$.

Proof of Theorem 1.1. We prove first that $b \ln a<4 \pi /(\alpha-1)$ is necessary. To this end, we observe that obviously $b \ln a \leq 4 \pi /(\alpha-1)$ is necessary since for $b \ln a>4 \pi /(\alpha-1), g \in A_{\alpha}(D)$. Assume therefore that $b \ln a=4 \pi /(\alpha-1)$ and that $\Gamma(a, b)$ is a set of sampling. By Theorem 5.1 of [10], there exists then a $\delta_{0}>0$ such that $\left\{\zeta_{m n}\right\}$ is a set of sampling whenever $\rho\left(z_{m n}, \zeta_{m n}\right) \leq \delta$. Thus there exists a $\delta_{1}>0$ such that the points $\zeta_{m n}=a^{m}\left(b n+i\left(1-\delta_{1}\right)\right)$ constitute a set of sampling. But this is equivalent to saying that $\Gamma\left(a,\left(1-\delta_{1}\right)^{-1} b\right)$ is a set of sampling, which is a contradiction by the first observation.

We prove next the sufficiency. We note that the right inequality of (7) is identical to (8) with $s=\alpha$ and therefore concentrate on proving the left inequality.

Let us for clarity write $\varepsilon=\beta-\frac{1}{2}(\alpha-1)$, which by assumption is positive. By Lemma 3.1, (4), (6), and the Cauchy-Schwarz inequality, we have

$$
\begin{aligned}
\left(1-|z|^{2}\right)^{\alpha}|f(z)|^{2} & \\
& \leq\left(\sum_{j}\left(1-\left|z_{j}\right|^{2}\right)^{\alpha / 2}\left|f\left(z_{j}\right)\right| \frac{\left(1-|z|^{2}\right)^{s+1 / 2-\varepsilon}\left(1-\left|z_{j}\right|^{2}\right)^{1 / 2+\varepsilon}}{\left|1-\overline{z_{j}} z\right|^{s+1}}\right)^{2} \\
& \leq \sum_{j}\left(1-\left|z_{j}\right|^{2}\right)^{\alpha}\left|f\left(z_{j}\right)\right|^{2} \frac{\left(1-\left|z_{j}\right|^{2}\right)^{\varepsilon}\left(1-|z|^{2}\right)^{2 s+1-2 \varepsilon}}{\left|1-\overline{z_{j}} z\right|^{s+1}} \sum_{k} \frac{\left(1-\left|z_{k}\right|^{2}\right)^{1+\varepsilon}}{\left|1-\overline{z_{k}} z\right|^{s+1}} .
\end{aligned}
$$

We choose $s>\varepsilon$ so that (12) applies to the last sum. Thus

$$
\left(1-|z|^{2}\right)^{\alpha}|f(z)|^{2} \leq C \sum_{j}\left(1-\left|z_{j}\right|^{2}\right)^{\alpha}\left|f\left(z_{j}\right)\right|^{2}\left(1-\left|z_{j}\right|\right)^{\varepsilon} \frac{\left(1-|z|^{2}\right)^{s+1-\varepsilon}}{\left|1-\overline{z_{j}} z\right|^{s+1}}
$$


Now integrating termwise with respect to $\mu$ and using (11), we get the desired estimate.

Proof of Theorem 1.2. To prove that $b \ln a>4 \pi /(\alpha-1)$ is necessary, we consider the problem of constructing a function $f \in A_{\alpha}(U)$ for which $f\left(z_{m n}\right)=0$ except when $m=n=0$. By the same argument as used for proving that $\Gamma(a, b)$ is a set of uniqueness when $b \ln a=4 \pi /(\alpha-1)$, we find that there is no solution if $b \ln a \geq 4 \pi /(\alpha-1)$.

Assume now that $\varepsilon=\frac{1}{2}(\alpha-1)-\beta>0$. The interpolation problem is solved explicitly by the formula

$$
f(z)=\sum_{j} \frac{a_{j}}{g^{\prime}\left(z_{j}\right)} \frac{g(z)}{\left(z-z_{j}\right)}\left(\frac{1-\left|z_{j}\right|^{2}}{1-\overline{z_{j}} z}\right)^{s}
$$

where $s>\varepsilon$. By (4), (6), and the Cauchy-Schwarz inequality, we have

$$
\begin{aligned}
\left(1-|z|^{2}\right)^{\alpha}|f(z)|^{2} & \\
& \leq\left(\sum_{j}\left(1-\left|z_{j}\right|^{2}\right)^{\alpha / 2}\left|a_{j}\right| \frac{\left(1-\left|z_{j}\right|^{2}\right)^{s+1 / 2-\varepsilon}\left(1-|z|^{2}\right)^{1 / 2+\varepsilon}}{\left|1-\overline{z_{j}} z\right|^{s+1}}\right)^{2} \\
& \leq \sum_{j}\left(1-\left|z_{j}\right|^{2}\right)^{\alpha}\left|f\left(z_{j}\right)\right|^{2} \frac{\left(1-\left|z_{j}\right|^{2}\right)^{s-\varepsilon}\left(1-|z|^{2}\right)^{1+2 \varepsilon}}{\left|1-\overline{z_{j}} z\right|^{s+1}} \sum_{k} \frac{\left(1-\left|z_{k}\right|^{2}\right)^{s+1-\varepsilon}}{\left|1-\overline{z_{k}} z\right|^{s+1}} .
\end{aligned}
$$

We apply (12) to the last sum, yielding

$$
\left(1-|z|^{2}\right)^{\alpha}|f(z)|^{2} \leq C \sum_{j}\left(1-\left|z_{j}\right|^{2}\right)^{\alpha}\left|f\left(z_{j}\right)\right|^{2}\left(1-\left|z_{j}\right|\right)^{s-\varepsilon} \frac{\left(1-|z|^{2}\right)^{1+\varepsilon}}{\left|1-\overline{z_{j}} z\right|^{s+1}}
$$

We integrate termwise with respect to $\mu$, use (11), and see that $f \in A_{\alpha}(D)$.

\section{REMARKS}

(A) The weighted Bergman space $A_{\alpha}(U)$ is the image of the Hardy space $H^{2}(\mathbf{R})$ under the wavelet transform,

$$
s(t) \mapsto y^{-1 / 2} \int_{-\infty}^{\infty} s(t) \overline{g((t-x) / y)} d t,
$$

with analyzing wavelet

$$
g(t)=(t+i)^{-(\alpha+1) / 2}
$$

[5]. $A_{\alpha}(U)$, therefore, can be considered as a time-scale representation of signals. Most notably, by the same transform, $A_{\alpha}(U)$ yields a phase space representation of quantum mechanics on the half-line, relevant in particular for the hydrogen atom; see Paul's part of [8].

The $\Gamma(a, b)$ generate discrete wavelet transforms. A set of sampling $\Gamma(a, b)$, a term we have borrowed from [7], corresponds via the wavelet transform to a frame of wavelets. See [3] for a general discussion of wavelet frames and their applications in signal analysis.

(B) It is known from [2] that if, roughly speaking, a discrete set is sufficiently dense then it is a set of sampling for a given Bergman space. Similarly, a 
sufficiently sparse set will be a set of interpolation [9]. These results, however, give no hint about a possible critical density.

(C) With one exception (the proof of the necessity in Theorem 1.1), our method of proof applies to $L^{p}$ spaces with $0<p$. We have concentrated on the case $p=2$ for simplicity and because it is the most interesting with respect to the applications and physical interpretation that we have in mind.

(D) The essential ingredients in our approach are the estimates (4) and (5). They can be obtained for any (analytic) automorphic form with respect to some Fuchsian group with compact fundamental domain, and we have analogues of our theorems for zero-sets of such forms; the critical quantity then is the hyperbolic area of the fundamental domain divided by the number of zeros there.

What is more interesting is the general question of relating functions with growth properties (5) or (4) to sets of sampling or interpolation; see [6] for a description of zero-sets of functions satisfying (4). We believe this is important for the study of more general sets of sampling or interpolation. The following observation makes it clear that it is not just a question of adapting the ideas of [11]. When perturbing the set $\Gamma(a, b)$ in the first part of the proof of Theorem 1.1, we changed the critical quantity $b \ln a$. In [11], on the other hand, a key step consisted in observing that sets that can be viewed as "uniform perturbations" of each other are essentially equivalent when considering the sampling or interpolation problem.

Note added in proof. A general treatment of sets of sampling and interpolation for weighted Bergman spaces can be found in the author's paper "Beurling type density theorems in the unit disk," recently submitted for publication.

\section{ACKNOWLEDGMENT}

This work was done while the author was enjoying a stay at Institut MittagLeffler, Djursholm, Sweden, whose warm hospitality is gratefully acknowledged.

\section{REFERENCES}

1. A. Beurling, The collected works of Arne Beurling, Vol. 2 Harmonic Analysis (L. Carleson et al., eds.), Birkhäuser, Boston, MA, 1989.

2. R. R. Coifman and R. Rochberg, Representation theorems for holomorphic and harmonic functions in $L^{p}$, Astérisque 77 (1980), 11-66.

3. I. Daubechies, The wavelet transform, time-frequency localization and signal analysis, IEEE Trans. Inform. Theory 36 (1990), 961-1005.

4. I. Daubechies and A. Grossmann, Frames in the Bargmann space of entire functions, Comm. Pure Appl. Math. 41 (1988), 151-164.

5. A. Grossmann, J. Morlet, and T. Paul, Transforms related to square integrable group representations II. Examples, Ann. Inst. Henri Poincaré Phys. Théor. 45 (1986), 293-309.

6. B. Korenblum, An extension of the Nevanlinna theory, Acta Math. 135 (1975), 187-219.

7. H. J. Landau, Necessary density conditions for sampling and interpolation of certain entire functions, Acta Math. 117 (1967), 37-52.

8. T. Paul and K. Seip, Wavelets and quantum mechanics, Wavelets and Their Applications (G. Beylkin et al., eds.), Jones and Bartlett, Boston, MA, 1992, 303-321.

9. R. Rochberg, Interpolation by functions in Bergman spaces, Michigan Math. J. 29 (1982) 229-236. 
10. K. Seip, Reproducing formulas and double orthogonality in Bargmann and Bergman spaces, SIAM J. Math. Anal. 22 (1991), 856-876.

11. K. Seip and R. Wallstén, Sampling and interpolation in the Bargmann-Fock space, Report Institut Mittag-Leffler 1990/91.

12. K. Zhu, Operator theory in function spaces, Marcel Dekker, New York, 1990.

Division of Mathematical Sciences, University of Trondheim, N-7034 Trondheim, NORWAY

E-mail address: seip@imf.unit.no 